Linköping studies in science and technology. Licentiate Thesis No. 1855

\title{
Low-rank optimization in system identification
}

Måns Klingspor 


\title{
Low-rank optimization in system identification
}

\author{
Måns Klingspor
}


This is a Swedish Licentiate's Thesis.

Swedish postgraduate education leads to a Doctor's degree and/or a Licentiate's degree.

A Doctor's Degree comprises 240 ECTS credits (4 years of full-time studies).

A Licentiate's degree comprises 120 ECTS credits,

of which at least 60 ECTS credits constitute a Licentiate's thesis.

Linköping studies in science and technology. Licentiate Thesis

No. 1855

Low-rank optimization in system identification:

Måns Klingspor

mans.klingsporeliu.se

www. control.isy.liu.se

Department of Electrical Engineering

Linköping University

SE-581 83 Linköping

Sweden

ISBN 978-91-7929-974-3ＩSSN 0280-7971

Copyright (C) 2019 Måns Klingspor

Printed by LiU-Tryck, Linköping, Sweden 2019 
"They call it Royale with Cheese." 



\section{Abstract}

In this thesis, the use of low-rank approximations in connection with problems in system identification is explored. Firstly, the motivation of using low-rank approximations in system identification is presented and the framework for lowrank optimization is derived. Secondly, three papers are presented where different problems in system identification are considered within the described lowrank framework. In paper $A$, a novel method involving the nuclear norm for estimating a Wiener model is introduced. As shown in the paper, this method performs better than existing methods in terms of finding an accurate model. In paper B and C, a group lasso framework is used to perform input selection in the model estimation which also is connected to the low rank framework. The model structures where these novel methods of input selection is used on are ARX models and state space models, respectively. As shown in the respective papers, these strategies of performing input selection perform better than existing methods in both terms of estimation and input selection. 



\section{Acknowledgments}

First of all, I would like to thank my supervisors Professor Anders Hansson and Associate professor Johan Löfberg for all the help, support and encouragement. Furthermore, I would like to thank Professor Michel Verhaegen and Doctor Reinier Doelman for productive collaboration which resulted in a conference paper as well as a journal article. Also, I would like to thank the Swedish Research Council whose funding has made this thesis possible.

This thesis has been substantially improved with the help from Angela Fontan, Oskar Ljungqvist, Fredrik Ljungberg, Erik Hedberg, Magnus Malmström and Gustaf Hendeby. Your help has been invaluable and I appreciate the time that you have spent on proofreading and general help with various issues and questions.

Finally, I would like to thank my family and foremost my beloved fiancée Amanda Nyberg.

Linköping, September 2019

Måns Klingspor 



\section{Contents}

\section{Background}

1 Introduction 3

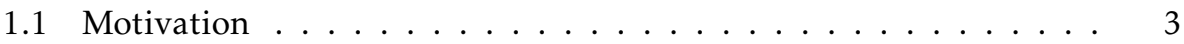

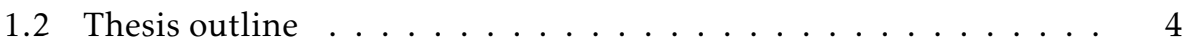

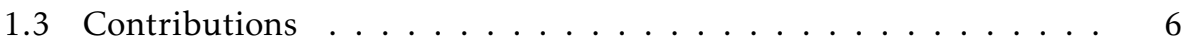

2 System identification $\quad 7$

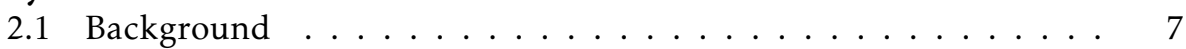

2.2 Model structures . . . . . . . . . . . . . . . . . 8

2.3 Low-rank framework in system identification . . . . . . . . . 11

2.3.1 A subspace method . . . . . . . . . . . . . . . 11

2.3.2 Input selection . . . . . . . . . . . . . . . 12

2.3.3 Bilinear matrix equalities ............ 13

3 Low-rank optimization $\quad 17$

3.1 The Rank Minimization Problem . . . . . . . . . . . . . . . 17

3.2 Mathematical framework . . . . . . . . . . . . . . . 18

3.3 Heuristics for the RMP . . . . . . . . . . . . . . . 20

3.4 Implementation of heuristic ............. 23

4 Concluding remarks $\quad 27$

$\begin{array}{lr}\text { Bibliography } & 29\end{array}$

\section{Publications}

A Identification of the dynamics of time-varying phase aberrations from time histories of the point-spread function $\quad 35$

1 Introduction ................... 37 
1.1 Notation . . . . . . . . . . . . . . . . . . . 40

2 Problem description . . . . . . . . . . . . . . . . 40

2.1 Linear and quadratic approximations of the PSF for small phase aberrations . . . . . . . . . . . . 40

2.2 VAR models and the identification problem . . . . . . . 42

3 Blind identification from quadratic measurements . . . . . . . . 44

3.1 Reformulating (18) into a rank constrained problem . . . . 44

3.2 A convex heuristic for (27) . . . . . . . . . . . . . 45

4 Numerical experiments . . . . . . . . . . . . . . . . . 47

$4.1 \quad$ Experimental setting . . . . . . . . . . . . . 44 47

4.2 Alternative methods . . . . . . . . . . . . . . . 49

4.3 Performance measures . . . . . . . . . . . . . 50

4.4 Results and discussion . . . . . . . . . . . . 50

5 Conclusion and future research $\ldots \ldots \ldots \ldots \ldots$

A The matrices in Equation (38) . . . . . . . . . . . . 53

B Settings of nonlinear solver . . . . . . . . . . . . . . 53

Bibliography ...................... 54

B Input selection in ARX model estimation using group lasso regularization $\quad 57$

1 Introduction . . . . . . . . . . . . . . . . . . 59

1.1 Notation . . . . . . . . . . . . . . . 60

2 Preliminaries..................... 60

2.1 ARMA models and ARX model estimation . . . . . . . . 60

3 Group lasso regularization . . . . . . . . . . . . . . . 63

4 Method ....................... 63

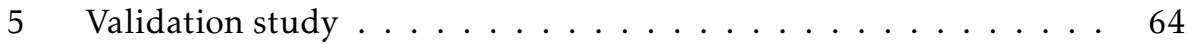

$5.1 \quad$ Preliminaries . . . . . . . . . . . . . . 64

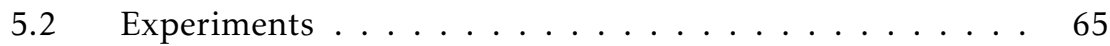

5.3 Results .................... 66

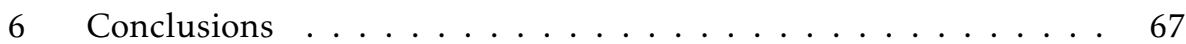

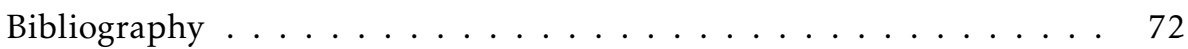

C Input selection in N2SID using group lasso regularization 73

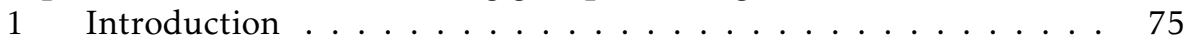

1.1 Notation . . . . . . . . . . . . . . . 76

2 Preliminaries.................... 77

2.1 State-space representation and input selection $\ldots \ldots . .77$

2.2 State-space identification with N2SID $\ldots \ldots \ldots \ldots$

3 Group lasso regularization . . . . . . . . . . . . . . 79

4 Method ..................... 80

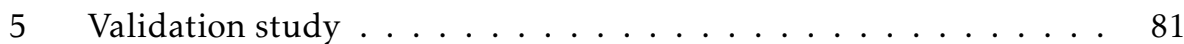

$5.1 \quad$ Preliminaries . . . . . . . . . . . . . . 81

5.2 Experiments . . . . . . . . . . . . . . . 83

5.3 Results .................... 84

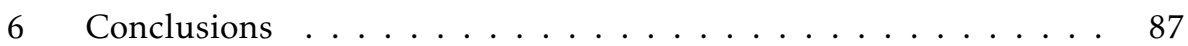


Bibliography ......................... 88 

Notation

Norms

\begin{tabular}{cl}
\hline Notation & Norm \\
\hline$\|\cdot\|_{1}$ & 1-norm \\
$\|\cdot\|_{2}$ & Euclidean norm or Spectral norm \\
$\|\cdot\|_{*}$ & Nuclear norm, Trace norm or Ky Fan norm \\
$\|\cdot\|_{F}$ & Frobenius norm \\
\hline
\end{tabular}

\section{Operations}

\begin{tabular}{cl}
\hline Notation & Definition \\
\hline$X \oplus Y$ & Direct sum of matrices $X$ and $Y$ \\
$X \odot Y$ & Hadamard product of matrices $X$ and $Y$ \\
\hline
\end{tabular}

\section{FUNCTIONS}

\begin{tabular}{cl}
\hline Abbreviation & Definition \\
\hline $\operatorname{rank}(X)$ & Rank of a (square) matrix $X$ \\
$\operatorname{tr}(X)$ & Trace of a (square) matrix $X$ \\
$\sigma(X)$ & Set of singular values of a matrix $X$ \\
$\lambda(X)$ & Set of eigenvalues of a (square) matrix $X$ \\
$X^{+}$ & Moore-Penrose inverse of a matrix $X$ \\
\hline
\end{tabular}




\begin{tabular}{cl}
\hline AbBREViations & \\
\hline Abbreviation & Definition \\
\hline ADMM & Alternating direction method of multipliers \\
ARX & Autoregressive exogenous model \\
BME & Bilinear matrix equality \\
RMP & Rank minimization problem \\
MIMO & Multiple-input and multiple output \\
MISO & Multiple-input and single-output \\
SDP & Semidefinite programming \\
SISO & Single-input and single-output \\
SVD & Singular value decomposition \\
\hline
\end{tabular}


Part I

Background 



\section{1}

\section{Introduction}

This chapter will provide a brief introduction to the research field of low-rank problems and its application in system identification. A motivating example will be provided as well as an example of an available strategy to solve this problem.

\subsection{Motivation}

One important field in science is system identification. In general, it deals with the construction of mathematical models of a system using observed data. Since there are different kinds of model structures, system identification is a large and diverse topic, however, the main idea is the same and it always involves the task to find the best possible model. What constitutes the best possible model might be ambiguous and depending on context. Intuitively, one might think that the best model is the one that best fits the observed data. However, there are good reasons why this is not always the case.

- Noisy data: If a model is trained to a noisy data set and solved exactly, this will yield a more complex model as it tries to explain the behavior of the noise. This is not justifiable nor possible since noise is by its nature random. Indeed, trying to explain the noise contained in the observed data is redundant as the noise will be completely different in future observations. With this background, there are clear incentives to adopt simpler model structures.

- Simplicity: Simple model structures that describe a data set are often favorable to more complex models. This is because simpler models can be both easier to use and to understand.

The key term here is simplicity. Since low-rank also in a sense implies simplicity, one can suspect that low-rank optimization can be employed to solve certain sys- 
tem identification problems.

One simple and concrete example is the identification of a discrete-time linear state space model:

$$
\begin{aligned}
x(t+1) & =A x(t)+B u(t) \\
y(t) & =C x(t)
\end{aligned}
$$

where $x(t) \in \mathbb{R}^{n}$ is the state vector, $u(t) \in \mathbb{R}^{p}$ is the input vector and $y(t) \in \mathbb{R}^{q}$ is the output vector which are represented as column vectors:

$$
x(t)=\left[\begin{array}{c}
x_{1}(t) \\
\vdots \\
x_{n}(t)
\end{array}\right], \quad u(t)=\left[\begin{array}{c}
u_{1}(t) \\
\vdots \\
u_{p}(t)
\end{array}\right], \quad y(t)=\left[\begin{array}{c}
y_{1}(t) \\
\vdots \\
y_{q}(t)
\end{array}\right]
$$

respectively. The goal is to estimate the matrices $A \in \mathbb{R}^{n \times n}, B \in \mathbb{R}^{n \times p}, C \in \mathbb{R}^{n \times q}$ so that the model fits the measurement data given by the data points $u(0), \ldots, u(N)$ and $y(0), \ldots, y(N)$. However, despite $p$ given inputs contained in the input vector $u(t)$, it is possible that not all of them actually affect the system output or that their effect is negligible. This will ideally correspond to zero columns in the estimated $B$ matrix, which clearly tells us which inputs can be discarded in the final model. Furthermore, more zero columns in $B$ yields a lower rank of the matrix. Thus, there is a clear connection between a system identification problem and low rank optimization. However, is it necessary to employ low-rank methods in order to solve this problem? If it is the case that some inputs do not affect the system at all, then this should be clear in the estimated $B$ matrix when using for instance a subspace method, see Chapter 2. In an ideal world that would be the case but the problem is that measurement data often contains noise which will distort the estimation. Thus, columns that should be zero are nonzero and therefore redundant inputs might be incorrectly included in the final model.

This specific problem formulation is commonly known as input selection. Seemingly, this is a case where the exact solution provided by some methods may not be desirable. Instead, in order to promote zero columns in the $B$ matrix, a method that penalizes the size of each column of $B$ might be the preferred option, see more details about this in Chapter 2.

\subsection{Thesis outline}

This thesis is split into two distinct parts: the first part aims to summarize the work and the second part contains published papers.

Mainly, the work in the thesis is centered around system identification and specifically on model structures for which low-rank optimization can be beneficial to employ compared to other existing methods. The first part of the thesis will introduce the basics in system identification and provide some examples of when low-rank optimization might be utilized. Furthermore, since rank minimization is a nonconvex problem and is almost always intractable to solve exactly, 
alternative methods utilizing heuristics will be introduced. Here, nuclear norm heuristics is introduced as well as some background on the theory.

The second part contains the three published papers. A summary of each paper is given below:

\section{Paper A: Identification of the dynamics of time-varying phase aberrations from time histories of the point-spread function}

Reinier Doelman, Måns Klingspor, Anders Hansson, Johan Löfberg and Michel Verhaegen. Identification of the dynamics of time-varying phase aberrations from time histories of the point-spread function. In Journal of the Optical Society of America A, volume 36, issue 5, pages 809-817, 2019.

Abstract: To optimally compensate time-varying phase aberrations with adaptive optics (AO), a model of the dynamics of the aberrations is required to predict the phase aberration at the next time step. We model the time-varying behavior of the phase aberration, expressed in Zernike modes, by assuming that the temporal dynamics of the Zernike coefficients can be described by a vector-autoregressive (VAR) model. We propose an iterative method based on a convex heuristic for a rank constrained optimization problem, to jointly estimate the parameters of the VAR model and the Zernike coefficients from a time series of measurements of the point spread function (PSF) of the optical system. By assuming the phase aberration is small, the relation between aberration and PSF measurements can be approximated by a quadratic function. As such, our method is a blind identification method for linear dynamics in a stochastic Wiener system with a quadratic nonlinearity at the output and a phase retrieval method that uses a time-evolutionmodel constraint and a single image at every time step.

Paper B: Input selection in ARX model estimation using group lasso regularization

Måns Klingspor, Anders Hansson and Johan Löfberg. Input selection in ARX model estimation using group lasso regularization. 18th IFAC Symposium on System Identification (SYSID), Proceedings, IFAC papers online, pages 897-902, 2018.

Abstract: In system identification, input selection is a challenging problem. Since less complex models are desirable, non-relevant inputs should be methodically and correctly discarded before or under the estimation process. In this paper we investigate an input selection extension in least-squares ARX estimation and show that better model estimates are achieved compared to the least-square solution, in particular, for short batches of estimation data.

\section{Paper C: Input selection in N2SID using group lasso regularization}


Måns Klingspor, Anders Hansson, Johan Löfberg and Michel Verhaegen. Input selection in N2SID using group lasso regularization. IFAC 2017 World Congress, IFAC papers online, Volume 50, Issue 1, pages 9474-9479, 2017.

\begin{abstract}
Input selection is an important and oftentimes difficult challenge in system identification. In order to achieve less complex models, irrelevant inputs should be methodically and correctly discarded before or under the estimation process. In this paper we introduce a novel method of input selection that is carried out as a natural extension in a subspace method. We show that the method robustly and accurately performs input selection at various noise levels and that it provides good model estimates.
\end{abstract}

\title{
1.3 Contributions
}

The author present the following scientific contributions in this thesis and publications.

- A novel method of estimating the phase aberrations and the aberration dynamics from intensity measurements of a point-spread function where the phase aberration dynamics can be described with a vector-valued autoregressive (VAR) model. Presented in paper A.

Reinier Doelman and Michel Verhagen had the idea for the paper. Reinier Doelman and Måns Klingspor implemented the idea and co-wrote the paper. Michel Verhaegen, Anders Hansson and Johan Löfberg supervised.

- A novel method of estimating and performing input selection with an ARX model using group lasso presented in paper B.

Anders Hansson had the idea for the paper. Måns Klingspor implemented the idea and wrote the paper. Anders Hansson and Johan Löfberg supervised.

- An extension to the N2SID algorithm to perform input selection using group lasso presented in paper $\mathrm{C}$.

Anders Hansson had the idea for the paper. Måns Klingspor implemented the idea and wrote the paper. Anders Hansson and Johan Löfberg supervised. 


\section{2}

\section{System identification}

The research field of system identification is about the construction of models of dynamical systems based on collected input and output measurement data from the system. The aim of this chapter is to provide a very brief introduction to the basics of system identification.

\subsection{Background}

A general, dynamical system $\mathcal{S}$ with inputs and outputs can be represented as in Figure 2.1 where $y(t) \in \mathbb{R}^{q}, u(t) \in \mathbb{R}^{p}$ are the output and input vectors respec-

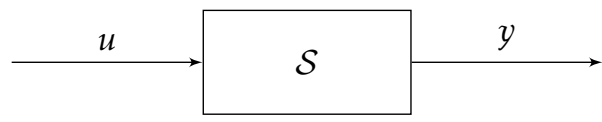

Figure 2.1: A system $\mathcal{S}$ with input signal $u(t)$ and output signal $y(t)$.

tively, $t \in \mathbb{R}_{+}$is the time and

$$
\mathcal{S}:\left\{\begin{array}{l}
\dot{x}(t)=f(t, x(t), u(t)) \\
y(t)=h(t, x(t), u(t))
\end{array}\right.
$$

is the mathematical description of the system where $x(t) \in \mathbb{R}^{n}$ are internal variables and $f: \mathbb{R}_{+} \times \mathbb{R}^{n} \times \mathbb{R}^{p} \rightarrow \mathbb{R}^{n}, g: \mathbb{R}_{+} \times \mathbb{R}^{n} \times \mathbb{R}^{p} \rightarrow \mathbb{R}^{q}$ are functions.

The main issue of system identification is that a mathematical description of $\mathcal{S}$ is unknown, and thus such a suitable description is sought using input and output data. Generally, the inputs of the system are known and the output data of the 
system is sampled uniformly. The set

$$
Z^{N}=\{u(1), \ldots, u(N), y(1), \ldots, y(N)\}
$$

contains sampled input and output signals at discrete times instances $t=1, \ldots, N$. Henceforth, we abuse the notation and $t$ is understood to be discrete-time instances. We denote a model with a specific structure $\mathcal{M}_{\theta}$ and the set of parameters $\theta$ and let

$$
\hat{y}(t \mid \theta)=\mathcal{M}_{\theta}\left(Z^{t-1}\right)
$$

be the predicted output at time $t$ simulating the model structure $\mathcal{M}_{\theta}$ using the input and output sequences from $Z^{t-1}$. With this, we define the prediction error

$$
e(t)=y(t)-\hat{y}(t \mid \theta)
$$

The objective is to minimize a function of the the prediction error

$$
V_{N}(\theta)=\sum_{t=1}^{N} \ell(e(t))
$$

where $\ell: \mathbb{R}^{q} \rightarrow \mathbb{R}_{+}$. For example $\ell$ can be given by $\ell(\cdot)=\|\cdot\|_{2}^{2}$. Then the parameter estimation is given by:

$$
\hat{\theta}=\underset{\theta}{\arg \min } V_{N}(\theta)=\underset{\theta}{\arg \min } \sum_{t=1}^{N}\|e(t)\|_{2}^{2},
$$

see $[13,18]$. Finally, for notational convenience and compactness the delay operator is defined:

Definition 2.1. Let $v(t)$ be any signal. Then the delay operator $q^{-T}$ is defined as

$$
q^{-T} v(t)=v(t-T)
$$

for $T \in \mathbb{Z}$.

\subsection{Model structures}

There are many different model structures in system identification [18]. Here we will provide some simple ones which are relevant for this thesis and also focus solely on discrete-time models. 


\section{Example 2.2}

A single-input single-output (SISO) autoregressive exogenous (ARX) model is given by the structure

$$
A(q) y(t)=B(q) u(t)+e(t)
$$

where

$$
\begin{aligned}
& A(q)=1+a_{1} q^{-1}+\ldots+a_{n_{a}} q^{-n_{a}} \\
& B(q)=b_{1} q^{-n_{k}}+\ldots+b_{n_{b}} q^{-n_{k}-n_{b}+1}
\end{aligned}
$$

are polynomials in $q^{-1}$ and $n_{a}, n_{b}, n_{k} \in \mathbb{Z}_{+}$. Note that this model structure can be easily extended into an Multiple Input Multiple Output model. In that case, $A(q)$ and $B(q)$ are matrices with polynomials as elements.

The ARX model is one of the most simple model structures. This simplicity is also reflected in the estimation, which corresponds to a convex optimization problem. Given collected data $Z^{N}$ from a system, let us derive the most basic method of estimation of an ARX model. First, we define

$$
\theta=\left[\begin{array}{llllll}
a_{1} & \ldots & a_{n_{a}} & b_{1} & \ldots & b_{n_{b}}
\end{array}\right]^{T}
$$

as the parameter vector, and define the vector $\varphi(t)$

$$
\varphi(t)=\left[\begin{array}{c}
-y(t-1) \\
\vdots \\
-y\left(t-n_{a}\right) \\
u\left(t-n_{k}\right) \\
\vdots \\
u\left(t-\left(n_{k}+n_{b}\right)+1\right)
\end{array}\right]
$$

Then, the ARX model can compactly be written as:

$$
y(t)=\theta^{T} \varphi(t)+e(t) .
$$

By stacking the data

$$
Y=\left[\begin{array}{c}
y(1) \\
\vdots \\
y(N)
\end{array}\right], \quad \Phi=\left[\begin{array}{c}
\varphi^{T}(1) \\
\vdots \\
\varphi^{T}(N)
\end{array}\right]
$$

the least-squares estimate of $\theta$ in Equation (2.5) is given by

$$
\hat{\theta}_{L S}=\underset{\theta}{\arg \min }\|Y-\Phi \theta\|_{2}^{2}
$$


which is a convex optimization problem. The solution can even be given in closed form. With the definition

$$
\begin{aligned}
f(\theta) & =\|Y-\Phi \theta\|_{2}^{2}=\left(Y^{T}-\theta^{T} \Phi^{T}\right)(Y-\Phi \theta) \\
& =Y^{T} Y-Y^{T} \Phi \theta-\theta \Phi^{T} Y+\theta^{T} \Phi^{T} \Phi \theta
\end{aligned}
$$

its gradient is given by

$$
\frac{\partial f}{\partial \theta}=2 \Phi^{T} \Phi \theta-2 \Phi^{T} Y
$$

If the matrix $\Phi^{T} \Phi$ has full rank, which is usually the case in practice if the inputs sequence is chosen sensibly, then $\left(\Phi^{T} \Phi\right)^{-1} \Phi^{T} Y$ is the only stationary point and because of convexity we can conclude that

$$
\theta_{L S}=\left(\Phi^{T} \Phi\right)^{-1} \Phi^{T} Y=\Phi^{+} Y
$$

where $\Phi^{+}=\left(\Phi^{T} \Phi\right)^{-1} \Phi^{T}$ denotes the Moore-Penrose inverse of $\Phi$ [12]. Note that this is one possible method of estimating the parameters of an ARX model. Arguably, it is the most straightforward method and easiest since the solution is on closed form. However, as previously discussed, there might be situations where other methods are more suitable.

In addition to input and output signals, models can also have internal variables which are known as states. These are used in state space models.

\section{Example 2.3}

A general discrete-time state space model is given by the structure

$$
\begin{aligned}
x(t+1) & =f(t, x(t), u(t), v(t)) \\
y(t) & =g(t, x(t), u(t), w(t))
\end{aligned}
$$

where $x(t) \in \mathbb{R}^{n}, u(t) \in \mathbb{R}^{p}, y(t) \in \mathbb{R}^{q}, f: \mathbb{R}_{+} \times \mathbb{R}^{n} \times \mathbb{R}^{p} \rightarrow \mathbb{R}^{n}$ and $g: \mathbb{R}_{+} \times \mathbb{R}^{n} \times \mathbb{R}^{p} \rightarrow$ $\mathbb{R}^{q}$ might be nonlinear functions. The vectors $v(t) \in \mathbb{R}^{n}$ and $w(t) \in \mathbb{R}^{q}$ are disturbances.

An important special case of this model structure is the discrete-time linear state space model:

$$
\begin{aligned}
x(t+1) & =A x(t)+B u(t)+v(t) \\
y(t) & =C x(t)+D u(t)+w(t)
\end{aligned}
$$

where $A \in \mathbb{R}^{n \times n}, B \in \mathbb{R}^{n \times p}, C \in \mathbb{R}^{n \times q}, D \in \mathbb{R}^{q \times p}$ are constant matrices.

The estimation of a linear state space model expressed as in Equation (2.19) is a well-studied problem in system identification [18]. Assume that we are given $Z^{N}$, that is, known inputs and output measurements. Certainly, if the state sequence $x(1), \ldots, x(N)$ is known, only the matrices $A, B, C, D$ need to be estimated. 
In that case, it is easy to see that the estimation problem can be formulated as a linear least squares problem as for the ARX model. However, normally the state sequence is unknown and the estimation problem can subsequently be formulated as

$$
\begin{aligned}
\underset{x, v, w, A, B, C, D}{\operatorname{minimize}} & \sum_{t=1}^{N}\|v(t)\|_{2}^{2}+\sum_{t=1}^{N}\|w(t)\|_{2}^{2} \\
\text { subject to } & x(t+1)=A x(t)+B u(t)+v(t) \\
& y(t)=C x(t)+D u(t)+w(t) .
\end{aligned}
$$

where $x(t)$ and $A, C$ are among the decision variables. Therefore, the constraints are in principle bilinear equalities. Thus, the problem in (2.20) is non-convex and applying for instance nonlinear least squares to solve the problem can be very sensitive to initialization. To circumvent this problem, so called subspace methods can be employed to solve the problem approximately [18]. In principle, these methods estimate the extended observability matrix

$$
O_{r}=\left[\begin{array}{c}
C \\
C A \\
\vdots \\
C A^{r-1}
\end{array}\right]
$$

for the system. When this matrix has been estimated, the state sequence can be constructed. Also, it is straight-forward to extract estimates for both $A$ and $C$ from $O_{r}$. Finally, once $\hat{A}$ and $\hat{C}$ are established, $B$ and $D$ can be estimated through a linear least squares problem. Usually, the number of states of the system $n$ is also unknown and one makes the assumption that there are $n<r$ states. Therefore, it is desirable that the estimation of $O_{r}$ is of low-rank in order to deduce the true $n$.

\subsection{Low-rank framework in system identification}

In this section, some examples of problems in system identification are provided where a low-rank framework may be beneficial to introduce.

\subsubsection{A subspace method}

Given the state space model

$$
\begin{aligned}
x(t+1) & =A x(t)+B u(t)+v(t) \\
y(t) & =C x(t)+D u(t)+w(t) .
\end{aligned}
$$

and measurement data $Z^{N}$, there are many different subspace methods available for estimation [18]. One particular method is the Nuclear Norm Subspace Identification, N2SID [25]. This methods works well for short data batches, that is, when $N$ is a relatively small number. As in other subspace methods, a key step is to 
estimate the extended observability matrix, $O_{r}$. Assume that we have the measurement data given above and let $r>n$ be an upper bound for the number of states. Here $n$ is the true but unknown number of states. Furthermore, we define the Hankel matrix

$$
U_{r}=\left[\begin{array}{cccc}
u(1) & u(2) & \ldots & u(N-r+1) \\
u(2) & u(3) & & \vdots \\
\vdots & & \ddots & \\
u(r) & u(s+1) & \ldots & u(N)
\end{array}\right]
$$

for the input $u(t)$ and define in the exact same manner Hankel matrices $Y_{r}$ and $V_{r}$ for $y(t)$ and $v(t)$ respectively. Furthermore, we define the Toeplitz matrix

$$
T_{u, r}=\left[\begin{array}{cccc}
D & 0 & \ldots & 0 \\
C B & D & & 0 \\
\vdots & & \ddots & \\
C A^{r-2} B & & \ldots & D
\end{array}\right]
$$

from the quadruple of system matrices $\{A, B, C, D\}$ and define $T_{y, r}$ from the quadruple $\{A, I, C, 0\}$ in the same manner. Finally, let

$$
X=\left[\begin{array}{llll}
x(1) & x(2) & \ldots & x(N-r+1)
\end{array}\right]
$$

be the true state sequence. These definitions yield the data equation:

$$
Y_{r}=O_{r} X+T_{u, r} U_{r}+T_{y, r} Y_{r}+E_{r}
$$

and a slight reformulation of this equation is to let $\hat{y}(t)=y(t)-e(k)$ and $\hat{Y}_{r}$ be a Hankel matrix defined in the same manner as $Y_{r}$ for $y(t)$. With the knowledge that $O_{r}$ should be of low rank since $r>n$, this yields the minimization problem

$$
\underset{\hat{Y}_{r} \in \mathcal{H}^{p}, \hat{T}_{u, r} \in \mathcal{T} p, m, \hat{T}_{y, r} \in \mathcal{T}}{\operatorname{minimp}} \operatorname{rank}\left(\hat{Y}_{r}-\hat{T}_{u, r} U_{r}-\hat{T}_{y, r} Y_{r}\right)
$$

where $\mathcal{T}^{p, m}$ denotes the class of lower triangular block-Toeplitz matrices with block entries $p \times m$ matrices and $\mathcal{H}^{p}$ denotes the class of block-Hankel matrices with block entries of $p$ column vectors.

\subsubsection{Input selection}

In the case of an excessive amount of inputs that do not affect the system and noise present in the measurements, performing so called input selection can yield a better model [15]. For instance, suppose that we have a MISO system with input signal $u_{1}(t), \ldots, u_{p}(t)$ and a scalar output signal $y(t) \in \mathbb{R}$. A possible model structure to use is the ARX model

$$
A(q) y(t)=B(q) u(t)+e(t)
$$


where $A(q)$ is a polynomial in $q^{-1}$ and $B(q)$ is a vector where each element is a polynomial in $q^{-1}$. The parameter vector becomes

$$
\theta=\left[\begin{array}{llllllllll}
a_{1} & \ldots & a_{n_{a}} & b_{1}^{1} & \ldots & b_{n_{b}^{1}}^{1} & \ldots & b_{1}^{p} & \ldots & b_{n_{b}^{p}}^{p}
\end{array}\right]^{T}
$$

and if one particular input $u_{m}(t), 1 \leq m \leq p$ does not affect the system, we should ideally have that $b_{1}^{m}=b_{2}^{m}=\ldots=b_{n_{b}^{p}}^{m}=0$. Stacking the input parameters in a matrix

$$
\Theta=\left[\begin{array}{ccc}
b_{n_{b}^{1}}^{1} & \ldots & b_{1}^{p} \\
\vdots & \vdots & \vdots \\
b_{1}^{1} & \ldots & b_{n_{b}^{p}}^{p}
\end{array}\right]
$$

it is thus desirable to achieve zero columns to remove redundant inputs. This is certainly connected to sparsity and thus lowering the rank of $\Theta$. It should however be noted that while a rank constraint might achieve this, it is not guaranteed. For example, the matrices

$$
\left[\begin{array}{ll}
1 & 0 \\
1 & 0
\end{array}\right],\left[\begin{array}{ll}
1 & 1 \\
1 & 1
\end{array}\right]
$$

obviously have the same rank. To remedy this problem, it is possible to instead employ a regression method such as a lasso [23]. In principle, a lasso method imposes a norm penalty on each variable which promotes zeros in the solution. In particular, when the variables have a natural grouping a group lasso can be used [9]. For example, in Equation (2.30) there is clearly a natural grouping among the variables because it is desirable to achieve zero columns. The natural group lasso penalty term is then given by

$$
\lambda \sum_{k=1}^{p}\left\|b^{k}\right\|_{2}
$$

where $\lambda \in \mathbb{R}_{+}$is a penalty parameter and $b^{k}, 1 \leq k \leq p$ is each column of $\Theta$.

\subsubsection{Bilinear matrix equalities}

Assume that we have the model structure given by

$$
\begin{aligned}
& x(t)=A x(t-1)+v(t) \\
& y(t)=x^{2}(t)+w(t)
\end{aligned}
$$

where $x(t) \in \mathbb{R}, A \in \mathbb{R}, v(t), w(t) \in \mathbb{R}$ are small disturbances. This model structure is known as a Wiener system where there is a nonlinearity in the outputs [27]. Because of this nonlinearity, it is not possible to employ a subspace method. For 
the times $t=1, \ldots, N$ and known measurements $u(1), \ldots, u(N), y(1), \ldots, y(N)$ the model estimation can be formulated as

$$
\begin{array}{cl}
\underset{x, v, w, A, B}{\operatorname{minimize}} & \sum_{t=1}^{N} v(t)^{2}+\sum_{t=1}^{N} w(t)^{2} \\
\text { subject to } & x(t)=A x(t-1)+v(t) \\
& y(t)=x^{2}(t)+w(t) .
\end{array}
$$

Since it is a scalar system we can write it on matrix form very easily. The state equations are given by

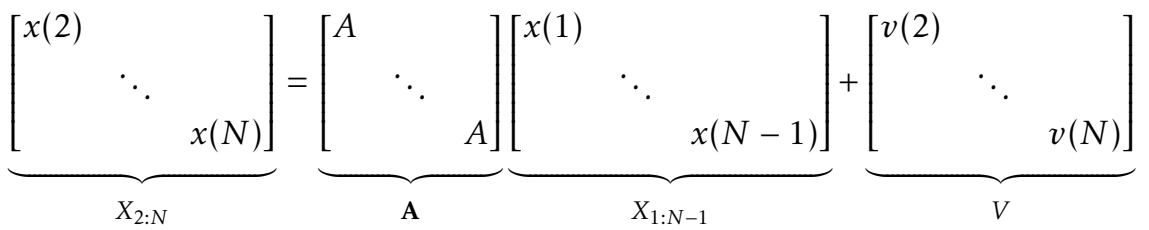

and the output equations by

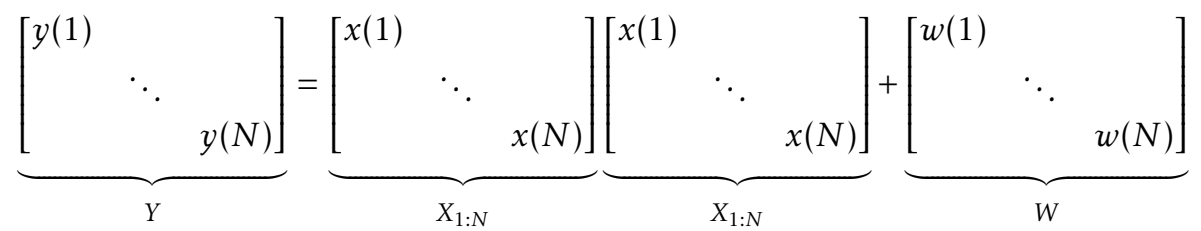

Then the equality constraint in Equation (2.34) can be written as

$$
\underbrace{\left[\begin{array}{cc}
X_{2: N}-V & 0 \\
0 & Y-W
\end{array}\right]}_{C}=\underbrace{\left[\begin{array}{cc}
\mathbf{A} & 0 \\
0 & X_{1: N}
\end{array}\right]}_{A} \underbrace{\left[\begin{array}{cc}
X_{1: N-1} & 0 \\
0 & X_{1: N}
\end{array}\right]}_{B}
$$

which is on the form $C=A P B(P=I)$. It is a special case of the bilinear matrix inequalities addressed in [10]. With this reformulation, we may write the problem as:

$$
\begin{array}{ll}
\underset{\mathcal{X}, A, V, W}{\operatorname{minimize}} & \|V\|_{F}^{2}+\|W\|_{F}^{2} \\
\text { subject to } & {\left[\begin{array}{cc}
X_{2: N}-V & 0 \\
0 & Y-W
\end{array}\right]=\left[\begin{array}{cc}
\mathbf{A} & 0 \\
0 & X_{1: N}
\end{array}\right]\left[\begin{array}{cc}
X_{1: N-1} & 0 \\
0 & X_{1: N}
\end{array}\right]}
\end{array}
$$

This problem could be solved by for instance using the method of non-linear least squares [20] which has the advantage of computational efficiency. However, because the problem is non-convex, such a method is typically prone to local minimas. In our experiments it has been shown that convergence to a good solution is difficult unless the initial guess is very close to the optimal solution [7]. Therefore, it is paramount to find an alternative strategy to solve this problem. Now, it 
turns out a bilinear matrix equality may be rewritten as a linear rank constraint. With the definition

$$
L(A, P, B, C, \mathbf{X}, \mathbf{Y})=\left(\begin{array}{cc}
C+A P \mathbf{Y}+\mathbf{X} P B+\mathbf{X} P \mathbf{Y} & (A+\mathbf{X}) P \\
P(B+\mathbf{Y}) & P
\end{array}\right)
$$

it holds that

$$
\operatorname{rank} L(A, P, B, C, \mathbf{X}, \mathbf{Y})=\operatorname{rank} P \Longleftrightarrow A P B=C,
$$

see [6] for more details and proof. With this, Equation (2.38) can be rewritten as

$$
\begin{aligned}
\underset{\mathcal{X}, A, V, W, \mathbf{X}, \mathbf{Y}}{\operatorname{minimize}} & \|V\|_{F}^{2}+\|W\|_{F}^{2} \\
\text { subject to } & \operatorname{rank} L(A, I, B, C, \mathbf{X}, \mathbf{Y})=\operatorname{rank} I
\end{aligned}
$$

and thus the bilinearity has been replaced with a linear rank constraint. Indeed, the problem in Equation (2.41) is also non-convex due to the rank constraint and in the next chapter it will be shown how rank constraints in general can be replaced with heuristics. 



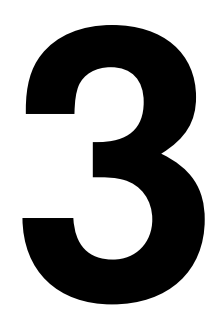

\section{Low-rank optimization}

\subsection{The Rank Minimization Problem}

As we have seen for some applications in system identification, minimizing ranks and searching for sparse solutions arise naturally. Of course, there are many other fields and applications where this is also the case, for example statistics, image processing, controller design and big data management [8]. A concrete example of the latter is the famous Netflix problem [17]. In this problem, a data set provided by Netflix contained 480189 users and 17770 individual, rateable items and more than 100 millions ratings provided by aforementioned users. This data set can be put in a big matrix such in Equation (3.1) called the ratings matrix. In this matrix, each user is represented by a column and each row contains the users' ratings for respective movie. As is well-known, the possible ratings are 1, 2, 3, 4 and 5 where a higher number corresponds to a better rating. If an item has not been rated by a user, its position is left void and marked symbolically with an asterisk.

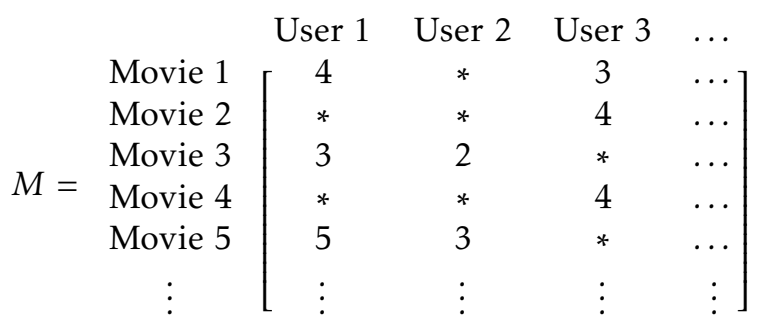

Since every user has, obviously, not rated nor watched every item available on Netflix, the objective is to fill in the gaps in the ratings matrix. This is to provide as accurate recommendations to users as possible. At the same time, the ratings matrix is expected to be low-rank since the user preferences oftentimes 
are described by a few parameters. Thus, a way to pose the problem is:

$$
\begin{array}{cl}
\underset{X}{\operatorname{minimize}} & \operatorname{rank}(X) \\
\text { subject to } & X_{i j}=M_{i j}, \quad(i, j) \in \mathcal{M} \\
& 1 \leq X_{i j} \leq 5, \quad(i, j) \notin \mathcal{M}
\end{array}
$$

where $\mathcal{M}$ is the set of indices of $M$ such that the element $(i, j)$ is not void. Of course, because of the non-convex structure of this problem it is very difficult to solve.

Now, looking at more general problems, one of the most basic low rank problems is given by

$$
\begin{array}{ll}
\underset{X}{\operatorname{minimize}} & \operatorname{rank}(X) \\
\text { subject to } & \frac{1}{2}\left\|X-X_{0}\right\| \leq \varepsilon
\end{array}
$$

where $X, X_{0} \in \mathbb{R}^{m \times n}$ and $\varepsilon \in \mathbb{R}_{+}$. Contrary to most low-rank problems, the solution is analytically and easily extracted by using the singular value decomposition of $X_{0}$ [8]. A related and more general problem is the matrix rank minimization problem (RMP) is given by

$$
\begin{array}{ll}
\text { minimize } & \operatorname{rank}(X) \\
\text { subject to } & X \in \mathcal{C}
\end{array}
$$

where $X \in \mathbb{R}^{m \times n}$ and $\mathcal{C} \subset \mathbb{R}^{m \times n}$ is a set denoting the constraints. Note that this is on the very same form as the Netflix problem posed in Equation (3.2). In various applications, $\mathcal{C}$ is a convex set. However, since the rank function is non-convex, the RMP is in itself a non-convex optimization problem [11]. Also, in addition to the non-convex nature of the RMP, it is also known to be NP-hard in the general case [24]. Therefore, heuristics must be employed in the general case in order to find an approximate solution. Before such heuristics is presented, some mathematical concepts required to do so is introduced in the next section.

\subsection{Mathematical framework}

First, we introduce the Singular value decomposition (SVD):

Theorem 3.1. Suppose $X \in \mathbb{R}^{m \times n}$. Then $X$ can be written as

$$
X=U \Sigma V^{T}
$$

where $U \in \mathbb{R}^{m \times m}$ is unitary, $\sum \in \mathbb{R}^{m \times n}$ is diagonal with non-negative numbers on the diagonal and $V \in \mathbb{R}^{n \times n}$ is unitary. The entries of $\sum$ are called singular values.

Proof: See [12].

An extension to the vector norms are the matrix norms defined below. 
Definition 3.2. (Matrix norm) Let $\|\cdot\|$ be a function such that $\|\cdot\|: \mathbb{R}^{m \times n} \rightarrow \mathbb{R}$. Let $\alpha \in \mathbb{R}$ be a scalar and $X, Y \in \mathbb{R}^{m \times n}$. Then $\|\cdot\|$ is a matrix norm if it satisfies the following properties:

(i) $\|\alpha X\|=|\alpha| \cdot\|X\|$

(ii) $\|X\| \geq 0$

(iii) $\|X\|=0 \Longleftrightarrow X=0$

(iv) $\|X+Y\| \leq\|X\|+\|Y\|$

Note: Additionally, some (but not all) matrix norms satisfy the property

$$
\|X Y\| \leq\|X\| \cdot\|Y\|
$$

for square matrices $X, Y \in \mathbb{R}^{n \times n}$.

There are different types of matrix norms, and we will focus on the Schatten norm. Definition 3.3. (Schatten norm) Let $X \in \mathbb{R}^{m \times n}$. Then the $p$-schatten norm of $X$ is

$$
\|X\|_{p}=\left(\sum_{i}^{\min \{m, n\}} \sigma_{i}^{p}(X)\right)^{1 / p}
$$

for $p \geq 1$.

Special cases of this norm include $p=2$. Assume that $X=U \Sigma V^{T}$ is the singular value decomposition of $X$. Then the 2-schatten norm of $X$ becomes

$$
\begin{aligned}
\sqrt{\sum_{i}^{\min \{m, n\}} \sigma_{i}^{2}(X)} & =\sqrt{\operatorname{tr}\left(\sum \Sigma^{T}\right)}=\sqrt{\operatorname{tr}\left(U^{T} U \Sigma \Sigma^{T}\right)}=\sqrt{\operatorname{tr}\left(U \Sigma V^{T} V \Sigma^{T} U^{T}\right)} \\
& =\sqrt{\operatorname{tr}\left(X X^{T}\right)}
\end{aligned}
$$

which corresponds to the Frobenius norm of $X$. Most important for this thesis is the case $p=1$ for which:

$$
\sum_{i}^{\min \{m, n\}} \sigma_{i}(X)=\operatorname{tr}(\Sigma)=\operatorname{tr}\left(\sqrt{\sum \Sigma^{T}}\right)=\operatorname{tr}\left(\sqrt{X X^{T}}\right)
$$

From this we define the nuclear norm:

Definition 3.4. (Nuclear norm) Let $X \in \mathbb{R}^{m \times n}$. Then

$$
\|X\|_{*}=\operatorname{tr}\left(\sqrt{X X^{T}}\right)=\sum_{i}^{\min \{m, n\}} \sigma_{i}(X)
$$

is called the nuclear norm of $X$ and is a matrix norm. 
Proof: Property (i) is easy to verify simply using the definition:

$$
\begin{aligned}
\|\alpha X\|_{*} & =\operatorname{tr}\left(\sqrt{(\alpha X)(\alpha X)^{T}}\right)=\operatorname{tr}\left(\sqrt{|\alpha|^{2} X X^{T}}\right)=\operatorname{tr}\left(|\alpha| \sqrt{X X^{T}}\right) \\
& =|\alpha| \operatorname{tr}\left(\sqrt{X X^{T}}\right)=|\alpha| \cdot\|X\|_{*}
\end{aligned}
$$

Property (ii) follows immediately since singular values are real and positive. Property (iii) has two directions. If $X=0$ then $\sigma_{1}, \ldots, \sigma_{n}=0$ which implies that $\|X\|_{*}=0$. If $\|X\|_{*}=0$, then $\sigma_{i}=0$ for all $i \in\{1, \ldots, \min \{m, n\}\}$ and $\Sigma=0$ which yields $X=U \Sigma V^{T}=0$ and proves Property (iii). Now, Property (iv) is a bit more tricky but it can be shown that

$$
\sup _{\sigma_{1}(Z) \leq 1} \operatorname{tr}\left(Z^{H} X\right)=\|X\|_{*}
$$

where $Z \in \mathbb{R}^{m \times n}[1]$. This yields

$$
\begin{aligned}
\|X+Y\|_{*} & =\sup _{\sigma_{1}(Z) \leq 1} \operatorname{tr}\left(Z^{H}(X+Y)\right) \leq \sup _{\sigma_{1}(Z) \leq 1} \operatorname{tr}\left(Z^{H} X\right)+\sup _{\sigma_{1}(Z) \leq 1} \operatorname{tr}\left(Z^{H} Y\right) \\
& =\|X\|_{*}+\|Y\|_{*}
\end{aligned}
$$

and we may conclude that the nuclear norm indeed is a matrix norm [1].

Finally, a remark about the connection between the rank of a matrix and its singular values should be made. Assume that $X=U \sum V^{T}$ and that its singular values are $\sigma_{1}, \ldots, \sigma_{r}, \sigma_{r+1}, \ldots, \sigma_{\min \{m, n\}}$ where $\sigma_{1}, \ldots, \sigma_{r}>0$ and $\sigma_{r+1}, \ldots, \sigma_{\min \{m, n\}}=0$. The singular value decomposition can be written as

$$
X=\sum_{i}^{\min \{m, n\}} u_{i} \sigma_{i} v_{i}^{T}
$$

that is, $X$ is a sum of rank-1 matrices where $u_{i}$ and $v_{i}$ are the $i$-th columns of $U$ and $V$, respectively. Since $\sigma_{1}, \ldots, \sigma_{r}>0, X$ is a sum of $r 1$-rank matrices. Hence, $\operatorname{rank}(X)=r$ which shows that there is a connection between the rank of a matrix and its amount of non-zero singular values.

\subsection{Heuristics for the RMP}

We return to the general RMP given by

$$
\begin{array}{ll}
\operatorname{minimize} & \operatorname{rank}(X) \\
\text { subject to } & X \in \mathcal{C}
\end{array}
$$

where $X \in \mathbb{R}^{m \times n}$ and $\mathcal{C} \subset \mathbb{R}^{m \times n}$ is a set denoting the constraints. As discussed in the previous section, it is intractable to solve this problem exactly, especially if it is of high dimension. It should be noted that there are attempts to solve this 
problem exactly, see for example $[19,21]$. Therefore, we need a viable heuristics for the RMP which will be derived here.

We begin by restricting ourselves to the case where $X \in \mathbb{R}^{n \times n}$ (square) and $X \geq 0$ (positive semidefinite). Of course, this implies that the eigenvalues of $X$ are real and nonnegative. Therefore, a reasonable heuristic for the RMP is to use

$$
\begin{array}{ll}
\operatorname{minimize} & \operatorname{tr}(X) \\
\text { subject to } & X \in \mathcal{C} \\
& X \geq 0
\end{array}
$$

since $\operatorname{tr}(X)=\sum_{k=1}^{n} \lambda_{i}(Z)=\|\lambda(X)\|_{1}$ where $\lambda(X) \in \mathbb{R}^{n}$ contains the eigenvalues of $X$. From this, we conclude that minimizing $\operatorname{tr}(X)$ is the same as minimizing the $\ell_{1}$-norm of a vector. Furthermore, it is a well-known result that minimizing the $\ell_{1}$-norm of a vector yields a sparse vector [8]. This was extensively shown in connection with Restricted isometry property, where the gap between practice and theory in using the $\ell_{1}$-norm to achieve sparse solutions was bridged [4]. In our case, this results in sparsity of $\lambda(X)$ and therefore several eigenvalues will be zero. Thus, $X$ will be of low-rank and we may conclude that this is a good heuristic in the sense that it achieves a low-rank solution which is also the goal for the RMP.

This result can be generalized for $X \in \mathbb{R}^{m \times n}$, that is, $X$ is not necessarily positive semidefinite or even square. For $X \in \mathbb{R}^{m \times n}$ it holds that rank $(X) \leq r$ if and only if there exists $U, V$ such that $\operatorname{rank}(U)+\operatorname{rank}(V) \leq 2 r$ and

$$
\left[\begin{array}{cc}
U & X \\
X^{T} & V
\end{array}\right] \geq 0,
$$

see [8]. If the above matrix is positive semidefinite, then $U$ and $V$ are that as well as they are on the diagonal. Thus, $U \oplus V \geq$ is also positive semidefinite. Therefore, in the same manner as before we may consider the problem:

$$
\begin{array}{cl}
\operatorname{minimize} & \frac{1}{2} \operatorname{tr}(U \oplus V) \\
\text { subject to } & {\left[\begin{array}{cc}
U & X \\
X^{T} & V
\end{array}\right] \geq 0} \\
& X \in \mathcal{C}
\end{array}
$$

where if $X \in \mathbb{R}^{m \times n}$, then $U \in \mathbb{R}^{n \times n}, V \in \mathbb{R}^{m \times m}$ are slack variables. The solution of this problem can be shown to be equal to the solution of the problem

$$
\begin{array}{ll}
\operatorname{minimize} & \|X\|_{*} \\
\text { subject to } & X \in \mathcal{C},
\end{array}
$$

see [8] for the proof. Therefore, the nuclear norm is a promising heuristic to employ in optimization problems that involve the rank function. Another reason why the nuclear norm is a good heuristic in this sense is because of the convex envelope, which is defined below. 
Definition 3.5. (Convex envelope) Assume that $f(X)$ is a non-convex function on the set $\mathcal{X}$ such that $f: \mathbb{R}^{m \times n} \rightarrow \mathbb{R}$. Then the convex envelope $g(x): \mathbb{R}^{m \times n} \rightarrow \mathbb{R}$ is the largest convex function such that

$$
g(X) \leq f(X)
$$

for all $X \in \mathcal{X}$.

For a concrete example of a convex envelope, see the sketch in Figure (3.1) where $f(x)$ is a non-convex function and $g(x)$ is its convex envelope. In this sketch,

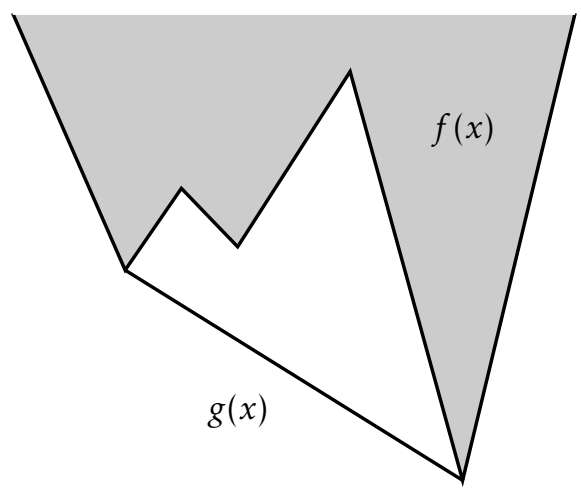

Figure 3.1: A non-convex function $f(x)$ and its convex envelope $g(x)$.

one can see that $f(x)$ and $g(x)$ share the same global minima, which is not a coincidence. In fact, if $\mathcal{X}=\mathcal{C}$ is a convex set and $g(x)$ is the convex envelope of $f(x)$ on $\mathcal{C}$ and

$$
\begin{aligned}
f^{*}=\text { minimize } & f(X) \\
\text { subject to } & X \in \mathcal{C} \\
g^{*}=\text { minimize } & g(X) \\
\text { subject to } & X \in \mathcal{C},
\end{aligned}
$$

then it holds that $f^{*}=g^{*}$, see [5]. While convex envelopes might appear to be a universal remedy for non-convex optimization, it should be noted that it is in general equally hard to find the global minima of $f(x)$ as it is to find its convex envelope $g(x)$ [5]. However, a function that has a convex envelope on closed form in a certain set is the rank function. This is stated in the following theorem:

Theorem 3.6. On the set $\mathcal{X}=\left\{X \in \mathbb{R}^{m \times n} \mid\|X\|_{2} \leq 1\right\},\|X\|_{*}$ is the convex envelope of $\operatorname{rank}(X)$.

Proof: See [8].

It is easy to see why $\|X\|_{*}$ is the convex envelope only on the set $\mathcal{X}=\{X \in$ $\left.\mathbb{R}^{m \times n} \mid\|X\|_{2} \leq 1\right\}$. Consider for example $X=a I \in \mathbb{R}^{n \times n}$ where $a>1$. Then $\|X\|_{2}=$ 
$a \cdot n>1$ so $X \notin \mathcal{X}, \operatorname{rank}(X)=n$ and $\|X\|_{*}=a \cdot n$. Obviously, $\|X\|_{*}>\operatorname{rank}(X)$ in this case. Thus, the rank function which is non-convex is replaced with the best possible convex lower bound with the constraint that $X$ must be in the set $\mathcal{X}=\left\{X \in \mathbb{R}^{m \times n} \mid\|X\|_{2} \leq 1\right\}$. However, in practice it has been observed that the nuclear norm is a favorable heuristic also if the constraint $\|X\|_{2} \leq 1$ is dropped, simply [8].

\subsection{Implementation of heuristic}

As we have seen, the non-convex RMP can ultimately be replaced with a nuclear norm heuristic. Of course, even though the problem

$$
\begin{array}{ll}
\text { minimize } & \|X\|_{*} \\
\text { subject to } & X \in \mathcal{C}
\end{array}
$$

is convex in contrast to the RMP, it is still difficult to solve. Mainly, it is because the nuclear norm is not differentiable everywhere for general matrices, see [2]. For example, if $X \in \mathbb{R}$ then $\|X\|_{*}=|X|$ which is not differentiable at $X=0$. However, because of convexity it can be possible to use subgradients to solve the problem.

Definition 3.7. (Subgradient) Let $f: \mathbb{R}^{m \times n} \rightarrow \mathbb{R}$ be a convex function. Then $g$ is a subgradient at $x_{0}$ if

$$
f(x)-f\left(x_{0}\right) \geq g^{T} \cdot\left(x-x_{0}\right)
$$

for all $x$. The set of all subgradients of $f$ at $x_{0}$ is called the subdifferential of $f$ at $x_{0}$ and denoted $\partial f\left(x_{0}\right)$.

An important result that can be shown is that $x_{0}$ is a global minimizer of $f(x)$ if and only if $0 \in \partial f\left(x_{0}\right)$. Note that if $f(x)$ is differentiable at $x_{0}$, then it can be shown that $\partial f\left(x_{0}\right)=\left\{\nabla f\left(x_{0}\right)\right\}$. If $f(x)$ is differentiable everywhere, then $x_{0}$ is a global minimzer if and only if $0 \in \partial f\left(x_{0}\right)=\left\{\nabla f\left(x_{0}\right)\right\}$. This means that $\nabla f\left(x_{0}\right)=0$ if and only if $x_{0}$ is a global minimizer for the convex and differentiable function $f(x)$. Certainly, a special case of convex optimization with subgradients is when the objective function is smooth and the normal theory of smooth convex optimization applies [3].

\section{Example 3.8}

The convex function $f(x)=|x|$ is differentiable everywhere except at $x_{0}=0$. Subgradients at $x_{0}=0$ of $f$ are given by $g$ such that

$$
|x| \geq g \cdot x
$$

for all $x$, see the sketch in Figure 3.2. This holds for all $g \in[-1,1]$. Therefore, $\partial f\left(x_{0}\right)=[-1,1]$. Furthermore, easy to see that for all $x_{0}<0$ then $\partial f\left(x_{0}\right)=\{-1\}$ and $x_{0}>0$ then $\partial f\left(x_{0}\right)=\{1\}$. Finally, since $0 \in \partial f\left(x_{0}\right)=[-1,1]$, then $x_{0}=0$ is a global minimizer for $f(x)=|x|$. 


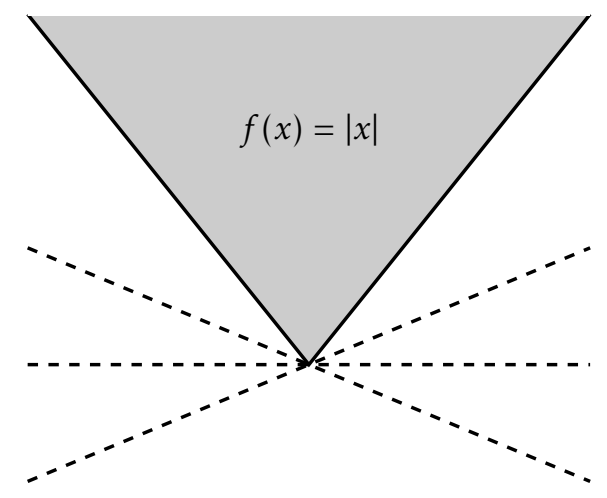

Figure 3.2: The function $f(x)=|x|$ and some of its subgradients.

It should be noted that convexity in a function does not necessarily imply the existence of a subgradient at every point in the domain. However, it exists for most well-behaved convex functions and is therefore a useful tool in non-smooth, convex optimization [3]. In particular, the function

$$
f(X)=\|X\|_{*}
$$

has the subdifferential

$$
\partial f(X)=U V^{T}+W
$$

where $X=U \Sigma V^{T}$ and $W$ is a matrix such that $U^{T} W=0, W V=0$ and $\|W\|_{2} \leq 1$ [26]. With this, it is straightforward to implement a subgradient method to solve the RMP see [14] for more details. While the subgradient method is the most general approach to solving non-smooth problems, it has fundamental problems with convergence. Namely, convergence is slow and is not in all cases controllable $[16,22]$. Therefore, it is more tractable to use the problem

$$
\begin{array}{cl}
\operatorname{minimize} & \frac{1}{2} \operatorname{tr}(U \oplus V) \\
\text { subject to } & {\left[\begin{array}{cc}
U & X \\
X^{T} & V
\end{array}\right] \geq 0} \\
& X \in \mathcal{C}
\end{array}
$$

which we have shown has the same solution as the RMP. This problem is a semidefinite program (SDP) which is a standard smooth, convex optimization problem and straightforward to implement. To solve an SDP, one can use for example interior-point methods [20]. Now, consider for example the RMP that appeared when estimating the extended observability matrix for linear state space model in (2.27):

$$
\underset{\hat{Y}_{r} \in \mathcal{H}^{p}, \hat{T}_{u, r} \in \mathcal{T}_{p, m}, \hat{T}_{y, r} \in \mathcal{T}^{p, p}}{\operatorname{minimize}} \operatorname{rank}\left(\hat{Y}-\hat{T}_{u, r} U_{r}-\hat{T}_{y, r} Y_{r}\right)
$$


Naturally, we can introduce the heuristics

$$
\underset{\hat{Y}_{r} \in \mathcal{H}^{p}, \hat{T}_{u, r} \in \mathcal{T}_{p, m}, \hat{T}_{y, r} \in \mathcal{T} p, p}{\operatorname{minimize}}\left\|\hat{Y}-\hat{T}_{u, r} U_{r}-\hat{T}_{y, r} Y_{r}\right\|_{*}
$$

which is convex and can be replaced with an SDP as discussed above.

Finally, consider the RMP that spawned from replacing a bilinearity with a linear rank constraint in Equation (2.41):

$$
\begin{aligned}
\underset{\mathcal{X}, A, V, W, \mathbf{X}, \mathbf{Y}}{\operatorname{minimize}} & \|V\|_{F}^{2}+\|W\|_{F}^{2} \\
\text { subject to } & \operatorname{rank} L(A, I, B, C, \mathbf{X}, \mathbf{Y})=\operatorname{rank} I
\end{aligned}
$$

In this case, it is replaced with the problem

$$
\underset{\mathcal{X}, A, V, W, \mathbf{X}, \mathbf{Y}}{\operatorname{minimize}}\|V\|_{F}^{2}+\|W\|_{F}^{2}+\lambda\|L(A, I, B, C, \mathbf{X}, \mathbf{Y})\|_{*}
$$

where $\lambda \in \mathbb{R}_{+}$is a regularization parameter [7].

As we will show in the papers, there are numerous advantages of utilizing this strategy of solving the aforementioned problems. Firstly, we acquire convex problems which are easy to implement because they can be replaced with SDPs. Secondly, and most importantly, they yield better results compared to existing methods. 



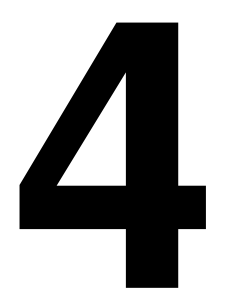

\section{Concluding remarks}

In this thesis, an introduction to system identification and some model structures have been provided. With these model structures, it has been shown situations where low-rank framework could possibly be employed in a beneficial manner. Mainly, this is the case when some kind of simplicity in the model structure is desirable to achieve. As has been shown, this includes the problem of input selection and finding the appropriate number of states in a state space model. Also, the low-rank framework appears, perhaps surprisingly, in estimating some specific Wiener model structures. However, as low-rank optimization is non-convex in its nature and thus not tractable, it was necessary to utilize heuristics. It has been shown that the nuclear norm in particular can serve as a suitable surrogate for the rank function. Finally, it has been shown how optimization involving the nuclear norm can be implemented as an SDP and thus easy to implement.

To conclude, the first part of the thesis should provide sufficient background for the second part where the published papers are provided. In these papers, the ideas of low-rank optimization and heuristics are implemented as novel methods for estimating certain model structures. Also, the performance of these methods are compared with current, commonly used methods and approaches. 



\section{Bibliography}

[1] Andersen Ang. Proving Ky Fan norm (nuclear norm) $\|X\|_{*}$ is a norm. https: / / angms.science/doc/LA/KyFanNorm.pdf, 2018. [Online; accessed 21-August-2019].

[2] Niclas Blomberg. On Nuclear Norm Regularization in System Identification. Licentiate thesis, Kungliga Tekniska Högskolan, 2016.

[3] Stephen Boyd and Lieven Vandenberghe. Subgradients. https:// see.stanford.edu/materials/lsocoee364b/01-subgradients_ notes.pdf, 2008. [Online; accessed 05-September-2019].

[4] E. J. Candes and T. Tao. Decoding by linear programming. IEEE Trans. Inf. Theor., 51(12):4203-4215, December 2005. ISSN 0018-9448. doi: 10.1109/TIT.2005.858979. URL http://dx.doi.org/10.1109/TIT. 2005.858979 .

[5] Du Ding-Zhu, P. M. Pardalos, and Wu Weili. Mathematical Theory of Optimization (Nonconvex Optimization and Its Applications). Springer-Verlag, Berlin, Heidelberg, 2006. ISBN 1402000154.

[6] Reinier Doelman and Michel Verhaegen. Sequential convex relaxation for convex optimization with bilinear matrix equalities. In Proceedings of the European Control Conference, 2016.

[7] Reinier Doelman, Måns Klingspor, Anders Hansson, Johan Löfberg, and Michel Verhaegen. Identification of the dynamics of time-varying phase aberrations from time histories of the point-spread function. J. Opt. Soc. Am. A, 36(5):809-817, May 2019. doi: 10.1364/JOSAA.36.000809.

[8] Maryam Fazel. Matrix Rank Minimization with Applications. PhD thesis, Stanford University, 2002.

[9] Jerome Friedman, Trevor Hastie, and Rob Tibshirani. A note on the group lasso and a sparse group lasso. arXiv:1001.0736, 22, 2010. 
[10] Jeremy G. VanAntwerp and Richard D. Braatz. A tutorial on linear and bilinear matrix inequalities. Journal of Process Control, 10:363-385, 082000. doi: 10.1016/S0959-1524(99)00056-6.

[11] Christian Grussler, Anders Rantzer, and Pontus Giselsson. Low-rank optimization with convex constraints. IRE Transactions on Automatic Control, 63(11):4000-4007, 2018. ISSN 0018-9286. doi: 10.1109/TAC.2018. 2813009.

[12] Roger A. Horn and Charles R. Johnson. Matrix Analysis. Cambridge University Press, 1990. ISBN 0521386322.

[13] K.J. Keesman. System Identification. Springer, 2011. ISBN 9780857295217.

[14] Krzysztof C. Kiwiel. Lagrangian relaxation via ballstep subgradient methods. Mathematics of Operations ResearchVol, 32:497-768, 2007.

[15] Måns Klingspor, Anders Hansson, Johan Löfberg, and Michel Verhaegen. Input selection in N2SID using group lasso regularization. In IFAC PAPERSONLINE, IFAC PAPERSONLINE, pages 9474-9479. ELSEVIER SCIENCE BV, 2017.

[16] Måns Klingspor, Anders Hansson, and Johan Löfberg. Input selection in arx model estimation using group lasso regularization. IFAC-PapersOnLine, 51: 897-902, 2018.

[17] Yehuda Koren, Robert Bell, and Chris Volinsky. Matrix factorization techniques for recommender systems, 2009.

[18] Lennart Ljung. System Identification: Theory for the User. Prentice Hall, Inc., second edition, 1992.

[19] Simone Naldi. Solving rank-constrained semidefinite programs in exact arithmetic. Journal of Symbolic Computation, 85, 07 2017. doi: 10.1016/j. jsc.2017.07.009.

[20] Jorge Nocedal and Stephen J. Wright. Numerical Optimization. Springer, second edition, 2006.

[21] Robert Orsi, Uwe Helmke, and John B. Moore. A newton-like method for solving rank constrained linear matrix inequalities. 2004 43rd IEEE Conference on Decision and Control (CDC) (IEEE Cat. No.04CH37601), 3:31383144, 2004.

[22] Naum Z. Shor. Minimization Methods for Non-Differentiable Functions. Springer-Verlag, 1979. ISBN 3540127631.

[23] Robert Tibshirani. Regression shrinkage and selection via the lasso. Journal of the Royal Statistical Society (Series B), 58:267-288, 1996.

[24] Lieven Vandenberghe and Stehphen Boyd. Semidefinite programming. SIAM Review, 1:49-95, 1996. 
[25] Michel Verhaegen and Anders Hansson. N2SID: Nuclear norm subspace identification of innovation models. Automatica, 72:57-63, 2016.

[26] Alistair Watson. Characterization of the subdifferential of some matrix norms. Linear Algebra and its Applications, 170:33-45, 1992.

[27] Adrian Wills and Lennart Ljung. Wiener System Identification Using the Maximum Likelihood Method. In Block-Oriented Nonlinear System Identification, pages 89-110. Springer, 2010. 



\section{Papers}

The papers associated with this thesis have been removed for copyright reasons. For more details about these see:

http://urn.kb.se/resolve?urn=urn:nbn:se:liu:diva-161286 


\section{DEPARTMENT OF ELECTRICAL ENGINEERING}

Linköping studies in science and technology. Licentiate Thesis No. 1855

Licentiate's Thesis.

Linköping University

SE-581 83 Linköping, Sweden

www.liu.se 